\title{
Investigation of CME dynamics in the LASCO field of view
}

\author{
A. Shanmugaraju ${ }^{1}$, Y.-J. Moon ${ }^{2}$, K.-S. Cho ${ }^{3}$, N. Gopalswamy ${ }^{4}$, and S. Umapathy ${ }^{5}$ \\ 1 Department of Physics, Arul Anandar College, Karumathur 625 514, India \\ e-mail: shanmugaraju_a@yahoo.com \\ 2 Department of Astronomy and Space Science, Kyung Hee University, Yongin 446-701, Korea \\ 3 Korea Astronomy and Space science Institute (KASI), Whaamdong, Yuseong-gu, Daejeon 305-348, Korea \\ 4 NASA Goddard Space Flight Center, Greenbelt, MD, USA \\ 5 School of Physics, Madurai Kamaraj University, Madurai 625021, India
}

Received 1 November 2007 / Accepted 14 March 2008

\section{ABSTRACT}

\begin{abstract}
Context. The speed-distance profile of CMEs is important for understanding the propagation of CMEs.
Aims. Our main aim is to study the initial speed of CMEs in the LASCO field of view and its role in subsequent CME propagation using the acceleration-speed profile. The secondary aim is to obtain the speed growth rate.

Methods. We considered the height-time data of 307 CMEs observed by SOHO/LASCO during January-March 2005. To study the CME speed profile, we used only 116 events for which there were at least 10 height-time measurements in the LASCO field of view. Using this data, we obtained their initial speed, extrapolated initial speed, and growth rate.

Results. The following results were found from this analysis. (i) The initial speed obtained from the first two data points is in the range $24-1208 \mathrm{~km} \mathrm{~s}^{-1}$, which is nearly similar to the range of linear speed $\left(67-920 \mathrm{~km} \mathrm{~s}^{-1}\right)$ obtained from a least squares fit through the entire h-t data set for each CME. (ii) However, the initial speed or extrapolated initial speed is much better correlated with acceleration and growth rate than the linear speed. (iii) Nearly two thirds of the events (74/116) are found to be accelerating. (iv) The speed growth rate is within the range -0.058 to $0.061 \times 10^{-3} \mathrm{~s}^{-1}$, and it decreases with the distance. (v) The final observed distance in the LASCO field of view depends very weakly upon the initial speed, or extrapolated initial speed whereas it depends strongly on the linear speed. The above results demonstrate the role played by the initial speed of the CMEs.
\end{abstract}

Key words. Sun: flares - Sun: coronal mass ejections

\section{Introduction}

It is now well-established that coronal mass ejections (CMEs) play an important role in causing geomagnetic disturbances (e.g., Gosling 1993). The CMEs are magnetized plasma ejected from the Sun into the solar corona and the solar wind. It has been found that the propagation of CMEs is controlled by the ambient solar wind plasma (Lindsay et al. 1999; Gopalswamy et al. 2000, 2001a,b). Gopalswamy (2000, 2001a) studied the CME acceleration over the Sun-Earth distance, while, Gopalswamy (2001b) considered acceleration within the LASCO field of view (FOV) for a set of shock-driving CMEs. The acceleration was positive for slow CMEs and negative for fast CMEs, which can be explained as the influence of an aerodynamic drag force on CME propagation (e.g., Vrsnak et al. 2001a,b, 2002, 2004; Cargill 2004).

Vrsnak et al. (2004) studied the kinematics of 5012 CMEs in the range $2-30 R_{\odot}$ observed during 1996 to 2001 . They analyzed the acceleration-speed $(a-v)$ relation and find a well-defined anticorrelation between the acceleration and speed of CMEs. They find that the majority of CMEs already or almost adjusted to the solar wind speed. Also they suggested that only CMEs (representing less than $10 \%$ of the CME population) with prolonged action of the propelling force attain a solar wind speed beyond $R>30_{\odot}$.

Yashiro et al. (2004) considered 6907 cataloged CMEs observed during 1996 to 2002 and shows that most of the slow CMEs $\left(v \leq 250 \mathrm{~km} \mathrm{~s}^{-1}\right)$ are accelerated; and the CMEs with intermediate speed $\left(250 \mathrm{~km} \mathrm{~s}^{-1}<v \leq 450 \mathrm{~km} \mathrm{~s}^{-1}\right)$ have little acceleration, whereas most of the fast CMEs $\left(v>450 \mathrm{~km} \mathrm{~s}^{-1}\right)$ are decelerated in the LASCO FOV. Because of this they suggest that the interaction between the CMEs and the solar wind is the important mechanism that determines CME trajectories in the LASCO FOV.

However, the radial evolution of CMEs in the LASCO FOV is not completely understood because of the residual acceleration and the acceleration of solar wind mainly below $10 R_{\odot}$. Also the interplay between various forces (Lorentz force, gravity, and the drag force) need to be examined to understand the dynamics of CMEs in this range. In contrast to the previous studies, we considered events that had many height-time measurements within the LASCO FOV. Moreover, in all the above studies, the linear speed (speed obtained by linear-fit on all the height-time measurements in the LASCO range) was taken as the representative speed of CMEs. However, in the present study, we considered the initial speed of the CMEs obtained from the first pair of height-time data. Since CMEs finish accelerating in the first 2-3 $R_{\odot}$, it is important to calculate CME properties close to the Sun (Wood et al. 1999; Zhang et al. 2001). This is our primary motivation in doing this study.

As suggested by Zhang \& Dere (2006), the first two phases of CME's kinematic evolution (initiation phase and acceleration phase) occur mainly in the inner corona, while the third phase (propagation phase) is largely in the outer corona. That is, many CMEs usually exhibit a more or less constant speed in the outer corona. Therefore, the speed of the CMEs in the inner corona, i.e., initial speed, is important for understanding the propagation phase in the outer corona. Also, the relatively constant speed of 
CMEs in the outer corona indicates that CME acceleration takes place mainly in the inner corona, where a strong but unstable magnetic field gives rise to the eruption. The initial speed is also important in deciding the formation of coronal shocks in the inner corona and generation of type II radio bursts. If the speed is greater than the alfven speed, it can form type II bursts in the metric wavelength domain (Cho et al. 2005; Lin et al. 2006).

The acceleration-speed $(a-v)$ profiles obtained by many authors show a large scatter mainly at CME speed $<1000 \mathrm{~km} \mathrm{~s}^{-1}$ (see e.g., Gopalswamy et al. 2000). We obtained the $a-v$ graphs using initial speed and compared them with those from linear speed, and discuss the role played by this initial speed. Also, we determined the growth rate and speed at zero acceleration at various heights from the $a-v$ graphs.

In the next section, we describe the data analysis and results. Discussions of the results are given in Sect. 3. Finally, conclusions are presented in Sect. 4.

\section{Data analysis and results}

We considered 307 CMEs observed by SOHO/LASCO during January-March 2005 as cataloged on the web site http:// cdaw.gsfc.nasa.gov/CME_list. Many results in the present paper were solely derived from the CME catalog. It is to be noted that the measurements used in LASCO are all sky-plane projected. The period January-March 2005 had reduced solar activity, so the chances of interaction between the CMEs would have been relatively small. For example, Gopalswamy et al. (2004) considered 23 interacting events, 5 of which occurred during solar minimum to rise phase, whereas 18 occurred during solar maximum phase. Based on the number of height-time measurements of these CMEs, we chose only those events with at least 10 height-time measurements in order to study the radial evolution of CMEs in the range $2-30 R_{\odot}$. It is known that, when the number of height measurements is large, the derived acceleration will have less error (Yashiro et al. 2004). Thus the sample reduced to 116 events, and we used this set for further analysis. From the SOHO/LASCO catalog, we utilized the linear speed, second-order initial speed, and acceleration of these CMEs. For each CME, we also used the cataloged values of several heights and times to obtain the initial speed, speed-distance $(v(h))$ profile, speed growth rate in the LASCO range. Our selection criterion (10 height-time measurements) biased the study to slow CMEs and hence positive acceleration. It should be stressed that there may be projection effects because cataloged values are projected heights, speeds, and accelerations.

In addition, a set of 39 events was separated from the 117 events that occurred beyond the 60 degree longitude. The locations of these events were identified from the SOHO/EIT images and eruptive prominences.

\subsection{Physical properties of CMEs}

First, we determined the initial speed of the CMEs in the LASCO FOV using the first two successive measurements of projected height $(h)$ and time $(t)$ mentioned for a particular event in the CME catalog as follows,

$v_{i}=(\delta h / \delta t)_{i}=\left(h_{i+1}-h_{i}\right) /\left(t_{i+1}-t_{i}\right)$

where $i=1$. We continued this process for other points $[i>1]$ in $h-t$ space and evaluated the instantaneous CME speed at various heights. The height corresponding to this speed $\left(v_{i}\right)$ was taken as $h=\left(h_{i}+h_{i+1}\right) / 2$.
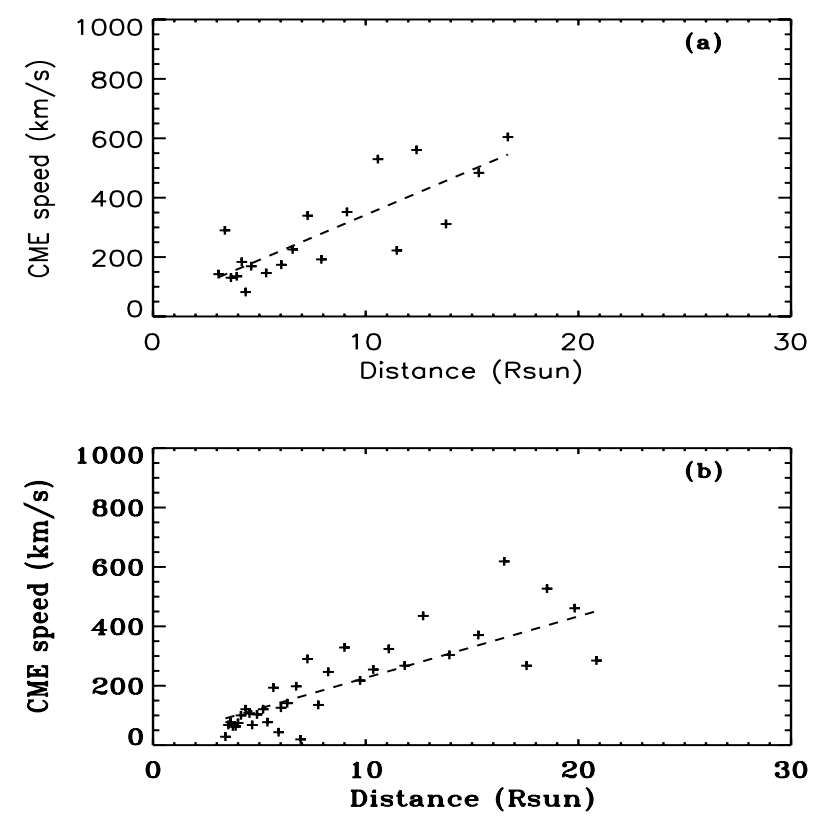

Fig. 1. a) Speed-distance profile of a CME observed on 8 January 2005. Dashed line shows the linear fit. b) same as above for the CME on 10 January 2005.

Second, the speed-distance profile, $v(h)$, was obtained for each event and then best-fitted with the straight line

$v(h)=m h+v_{\mathrm{o}}$

to obtain the slope (called as "growth rate", $m=\mathrm{d} v / \mathrm{d} h$ ) and constant (called "extrapolated initial speed" of the CME, $v_{\mathrm{o}}$ ).

The initial speed was calculated from the first pair of points in $h-t$ space as in Eq. (1), whereas the extrapolated initial speed $\left(v_{\mathrm{o}}\right)$ was obtained from the above linear equation (Eq. (2)) after least-square fitting. That is, we obtained $v_{\mathrm{o}}$ by setting $h=0$ in this equation. This differs from the definition of $h_{i}$ in the CME online catalog. The catalog measures $h_{i}$ from the Sun center. The $v(h)$ profiles given in the catalog were obtained by second-order polynomial fitting rather than successive differences that were performed in the present paper.

Finally, we compared the three speeds of CMEs (linear speed from the catalog, initial speed in the LASCO FOV, and the extrapolated initial speed derived in the present study) by correlating them with physical properties of CMEs such as acceleration and growth rate.

To illustrate the above analysis, the speed-distance profiles and the linear fit are shown in Figs. 1a and b for two CMEs ( 8 and 10 January 2005). For the CME on 8 January 2005, the initial speed, growth rate, and extrapolated initial speed are $143 \mathrm{~km} \mathrm{~s}^{-1}$, $0.0437 \mathrm{~s}^{-1}$, and $38 \mathrm{~km} \mathrm{~s}^{-1}$, respectively. The same parameters for the CME on 10 January 2005 are $28 \mathrm{~km} \mathrm{~s}^{-1}, 0.0297 \mathrm{~s}^{-1}$, and $19 \mathrm{~km} \mathrm{~s}^{-1}$, respectively. The CME speed increases with height, so the growth rate is positive for both these events. The linear speed of these two events reported in the catalog are 256 and $251 \mathrm{~km} \mathrm{~s}^{-1}$, respectively, which are entirely different from the initial speed; however, the cataloged initial speed due to quadratic fit was reported as $0 \mathrm{~km} \mathrm{~s}^{-1}$ in the online CME catalog for the above two events.

The distributions of the different speeds are shown in Fig. 2. Most of the events have speeds less than around $1000 \mathrm{~km} \mathrm{~s}^{-1}$. The linear speed ranges from $67-920 \mathrm{~km} \mathrm{~s}^{-1}$ (average $=387 \mathrm{~km} \mathrm{~s}^{-1}$ ), whereas the initial speed ranges from $24-1208 \mathrm{~km} \mathrm{~s}^{-1}$ (average $\left.=320 \mathrm{~km} \mathrm{~s}^{-1}\right)$. The initial speed is 

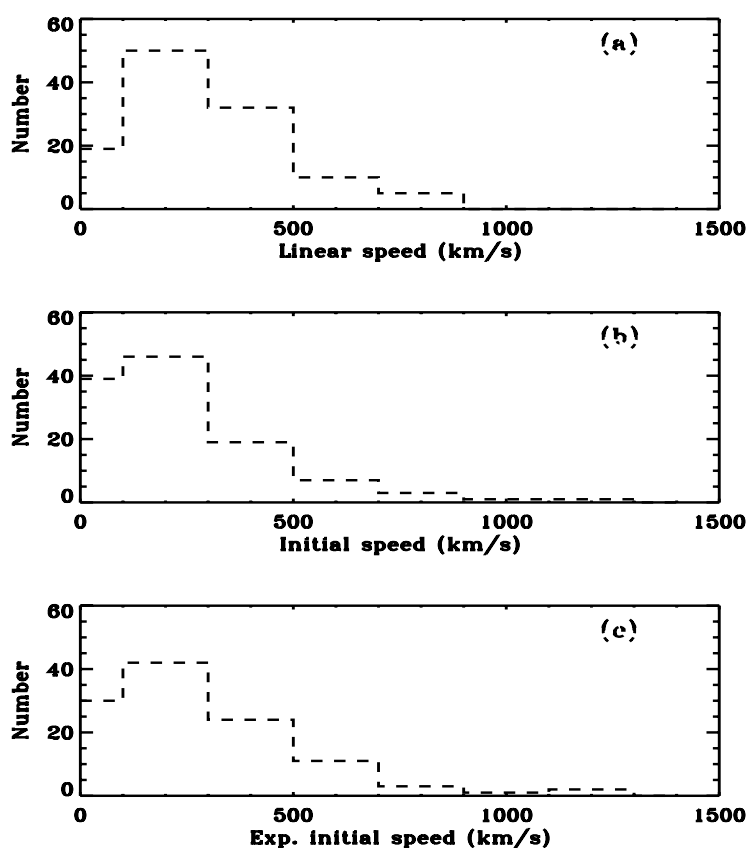

Fig. 2. Distributions of a) linear speed, b) initial speed, c) extrapolated initial speed. See text for details on speeds.

slightly less than the linear speed on average, confirming that the majority of the CMEs in our sample are accelerating. By limiting the range of both the distributions of initial speed and linear speed to $67-920 \mathrm{~km} \mathrm{~s}^{-1}$, we found that these average speeds do not change.

\subsection{Correlation between the properties}

We performed a correlation analysis between the CME speed and other CME properties. The linear speed and acceleration were taken from the online CME catalog. The scatter plots of linear speed with the acceleration and growth rate are presented in Figs. 3a, b. There is no correlation or very weak correlation between linear speed with acceleration and growth rate.

The same correlation analysis was done for initial speed and extrapolated initial speed of the CMEs with other properties and the results are represented in Figs. 4 and 5, respectively. One can see a better correlation $(r \sim 0.5)$ in Figs. 4a and b for initial speed with acceleration and growth rate than for the linear speed in Fig. 3. The correlation (not shown here) between the cataloged initial speed from quadratic fits with acceleration and growth rate were found to be comparable to the initial speed described in the last section. This in turn confirms the cataloged values of initial speed from quadratic fits. The correlations of the extrapolated initial speed (Fig. 5) are similar to those of the initial speed (Fig. 4), but a little higher ( $r \sim 0.6$, see Figs. 5a, b). In all the above cases, the second-order fit gave more or less similar correlation coefficients.

We also calculated the initial acceleration from the first two speed points and the time difference similar to the determination of initial speed as described in the previous section. Although the range of initial acceleration (minimum = $-15.7 \mathrm{~m} \mathrm{~s}^{-2}$, maximum $=18.13 \mathrm{~m} \mathrm{~s}^{-2}$, average $=0.91 \mathrm{~m} \mathrm{~s}^{-2}$ ) is similar to the range of acceleration (minimum $=-32.3 \mathrm{~m} \mathrm{~s}^{-2}$, maximum $=21.3 \mathrm{~m} \mathrm{~s}^{-2}$, average $=1.3 \mathrm{~m} \mathrm{~s}^{-2}$ ) reported in the catalog from a quadratic fit, there seems to be significant differences

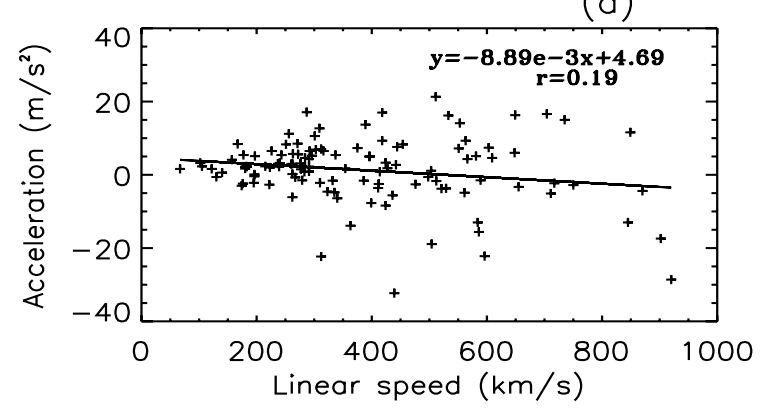

(b)

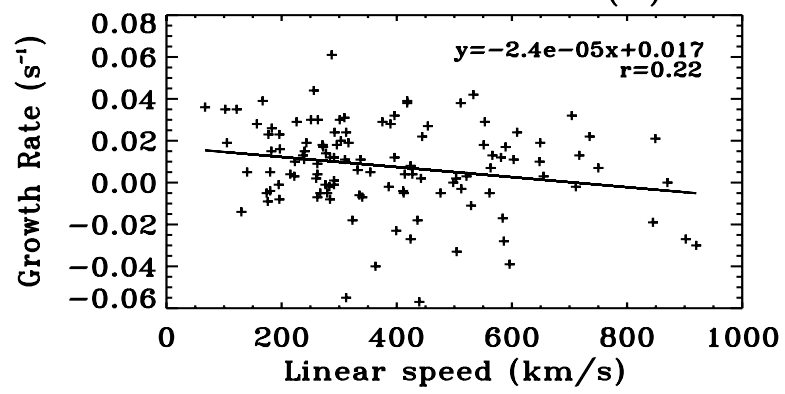

Fig. 3. Correlation between the linear speed with a) acceleration, b) growth rate.

(a)

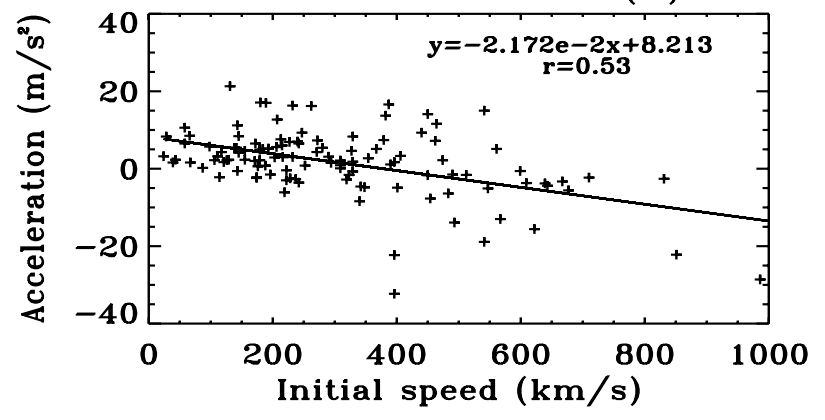

(b)

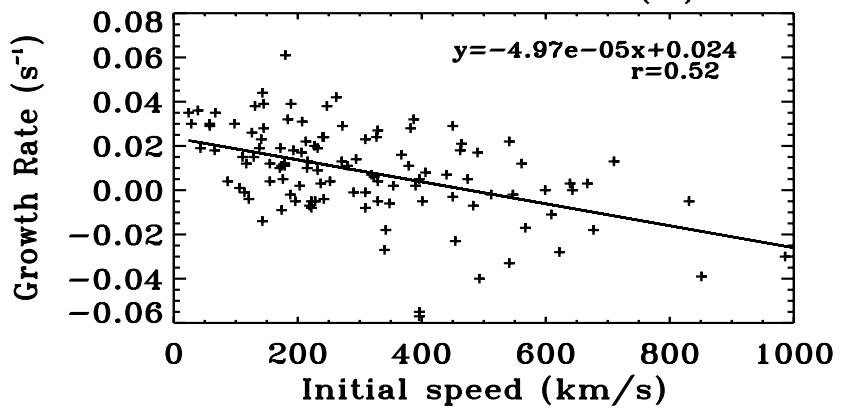

Fig. 4. Correlation between the initial speed with a) acceleration, b) growth rate.

in individual cases. There was no correlation between the initial acceleration and initial speed.

\section{Discussion}

\subsection{The physical properties of CMEs}

Since the linear speed of the CMEs in our sample is in the range $67-920 \mathrm{~km} \mathrm{~s}^{-1}$, CMEs faster than $920 \mathrm{~km} \mathrm{~s}^{-1}$ were automatically excluded by our selection criteria so that at least 


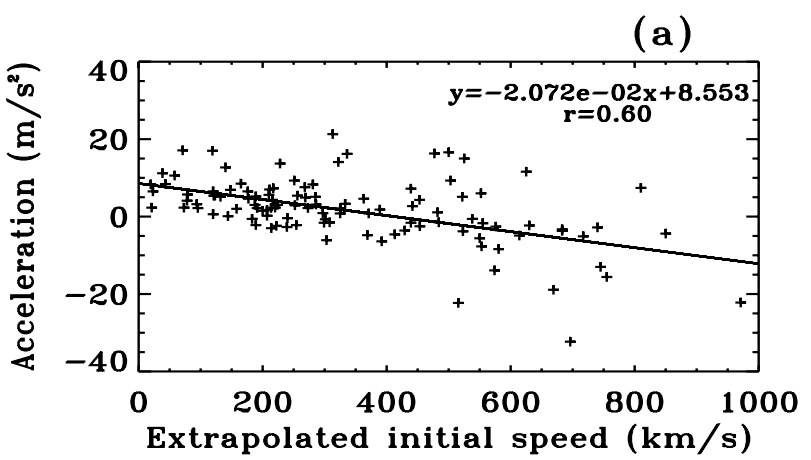

(b)

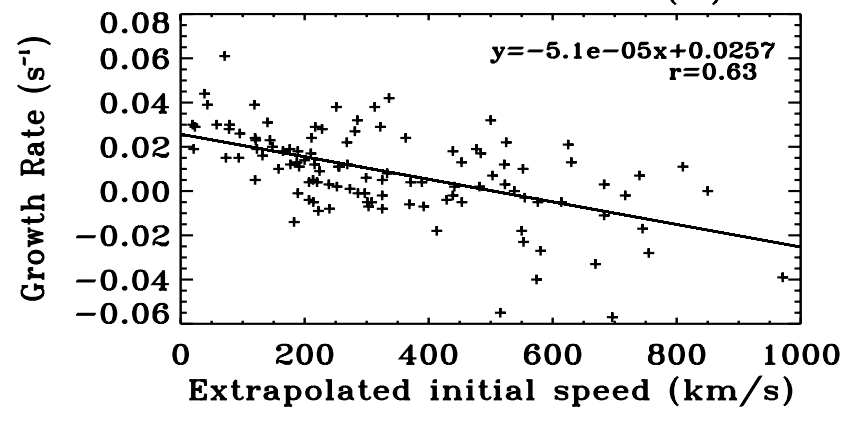

Fig. 5. Correlation between the extrapolated initial speed with a) acceleration and $\mathbf{b}$ ) growth rate.

10 height-time measurements were needed. When the events were split into three groups according to their linear speed $\left(v \leq 250 \mathrm{~km} \mathrm{~s}^{-1}, 250 \mathrm{~km} \mathrm{~s}^{-1}<v \leq 450 \mathrm{~km} \mathrm{~s}^{-1}, v>450 \mathrm{~km} \mathrm{~s}^{-1}\right)$, we got 27, 54, and 35 events in each category, respectively. There were more CMEs in the intermediate speed category. The average acceleration of CMEs in each category is $2.0 \mathrm{~m} \mathrm{~s}^{-2}$, $1.7 \mathrm{~m} \mathrm{~s}^{-2}$, and $-0.029 \mathrm{~m} \mathrm{~s}^{-2}$, respectively. It implies that they tend to be decelerated as the linear speed increases. This result obtained using events with a minimum of 10 points in $h-t$ space, agrees with that of earlier results (for example, Gopalswamy et al. 2000, 2001; Yashiro et al. 2004). Yashiro et al. (2004) used a larger data set of $6907 \mathrm{CMEs}$ out of which the acceleration of 3058 CMEs were measured. They considered events having a minimum of 5 height-time measurements.

On the other hand, the trend toward faster CMEs decelerating more was clearly seen when the CMEs were grouped according to their initial speeds in contrast to linear speeds. In this case, the average acceleration was estimated to be $4.4 \mathrm{~m} \mathrm{~s}^{-2}, 1.4 \mathrm{~m} \mathrm{~s}^{-2}$ and $-5.77 \mathrm{~m} \mathrm{~s}^{-2}$, respectively. Hence we stress on the importance of initial speed of CMEs in the LASCO range for further propagation of CMEs. When we grouped the CMEs according to their initial speed rather than the linear speed, we got 57, 33 and $26 \mathrm{CMEs}$ in each category. In this particular analysis, we got more CMEs with speed less than $250 \mathrm{~km} \mathrm{~s}^{-1}$. This may be because the speed changes over the LASCO FOV, so the average speed over the LASCO FOV is expected to be higher than the initial speed. We found that there were differences between the linear speed and initial speed as seen from the large scatter in Fig. 6. The percentage difference in several events was up to $50 \%$ or more.

There were more accelerating cases (74/116), because our sample was biased towards lower speeds (the maximum speed was limited to around $900 \mathrm{~km} \mathrm{~s}^{-1}$ by the selection criteria). Similarly, there were more cases with a positive growth rate $(\mathrm{d} v / \mathrm{d} h)$. Most of the fast CMEs $\left(>900 \mathrm{~km} \mathrm{~s}^{-1}\right)$ are decelerated in the LASCO FOV (Yashiro et al. 2004).

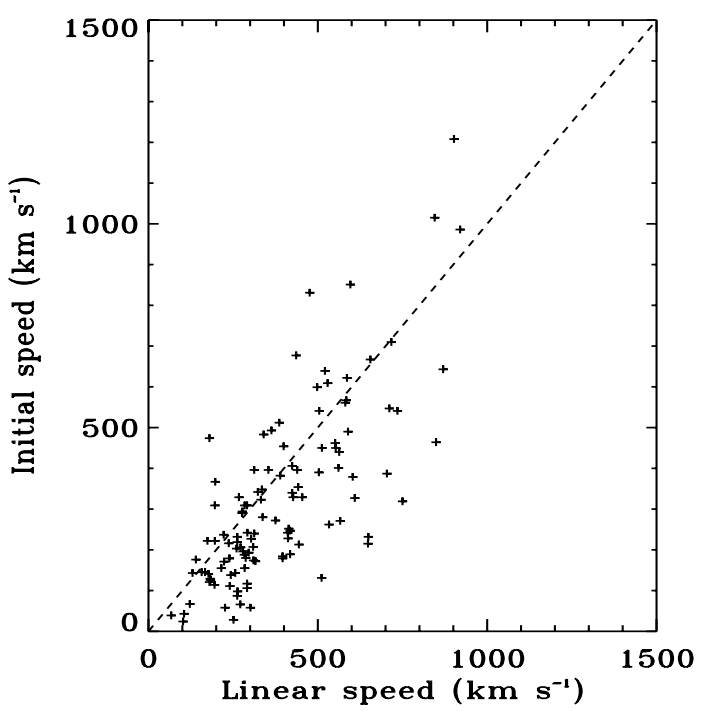

Fig. 6. Comparison between the initial speed and linear speed. The dashed line is shown for a perfect correlation.

The growth rate was found to be within the range -0.058 to $0.061 \times 10^{-3} \mathrm{~s}^{-1}$, which agrees with that of $\operatorname{Vrsnak}(2001 \mathrm{a}, \mathrm{b})$ for coronal transients. The term "growth rate" is the speed growth rate that was already used by other authors (e.g., Vrsnak 2001a,b) to describe the evolution of CME speed as a function of heliocentric distance. It is also important for understanding CME-associated phenomena such as type II radio bursts. The shock-driving capability of CMEs depends on the speed, so how the speed varies decides the shock formation. For example, if the speed increases rapidly above the Alfven speed, it can form type II bursts in the metric wavelength domain. If the speed increases slowly and become Alfvenic in the interplanetary medium, it can drive type II bursts in the kilometric wavelength region.

Dividing the 116 events into five groups using the mean radial distance $(R i+R f / 2, R i$, and $R f$ being the initial and final distance observed within LASCO FOV), we obtained the $a-v$ profiles in the five different ranges (5-7, 7-9, 9-11, 11-13, 13-15 $R_{\odot}$ ). From these $a-v$ profiles and regression analysis as described in Vrsnak et al. (2004), we determined the growth rate $(\gamma)$ at different heights. These values, along with the $x$-axis intercepts (speed at zero acceleration, $V_{0}$ ), are given in Table 1. The first three columns give the height range, number of CMEs in the particular range, and average of the mean radial distance of CMEs in each group; Cols. 4-6 give the values of $V_{0}, \gamma$, and the correlation coefficient of each $a-v$ graph. The variation in the growth rate with the mean radial distance is shown in Fig. 7. One can see the decreasing trend in the growth rate over the distance that is similar to the results of Vrsnak (2001a,b). This height dependency of the growth rate was confirmed in an alternative way as follows. When we examined the growth rate of individual CMEs as a function of their first observed distance in the LASCO FOV, the height dependency of growth rate was obtained. On the other hand, the $x$-axis intercepts $\left(V_{0}\right)$ increases with the height.

\subsection{Correlation analysis}

As seen in Sect. 2, the correlation of linear speed with acceleration and growth rate is weak. On the other hand, there is a better correlation between the initial or extrapolated initial 
Table 1. Growth rate $(\gamma)$ and speed at zero acceleration obtained for different sets of CMEs from the $a-v$ graphs $^{a}$.

\begin{tabular}{cccccccccccc}
\hline \hline Range & Number & Rmean & \multicolumn{3}{c}{ a-Vinitial } & \multicolumn{3}{c}{ a-Vlinear } & \multicolumn{3}{c}{ a-Vextrap } \\
\cline { 4 - 12 }$\left(R_{\odot}\right)$ & of CMEs & $\left(R_{\odot}\right)$ & $V_{0}$ & $\gamma\left(\times 10^{-3}\right)$ & $\mathrm{C}$ & $V_{0}$ & $\gamma\left(\times 10^{-3}\right)$ & $\mathrm{C}$ & $V_{0}$ & $\gamma\left(\times 10^{-3}\right)$ & $\mathrm{C}$ \\
\hline $5-7$ & 20 & 6.19 & 160 & 0.039 & 0.58 & 185 & 0.046 & 0.58 & 180 & 0.034 & 0.78 \\
$7-9$ & 27 & 7.88 & 330 & 0.037 & 0.63 & 410 & 0.017 & 0.2 & 350 & 0.039 & 0.76 \\
$9-11$ & 17 & 10.01 & 400 & 0.022 & 0.51 & 560 & 0.014 & 0.33 & 420 & 0.022 & 0.66 \\
$11-13$ & 19 & 11.95 & 490 & 0.022 & 0.59 & - & - & - & 510 & 0.022 & 0.59 \\
$13-15$ & 18 & 13.87 & 550 & 0.029 & 0.76 & 650 & 0.019 & 0.4 & 600 & 0.025 & 0.76 \\
\hline
\end{tabular}

${ }^{a}$ If there is a blank, it means the particular value cannot be measured.

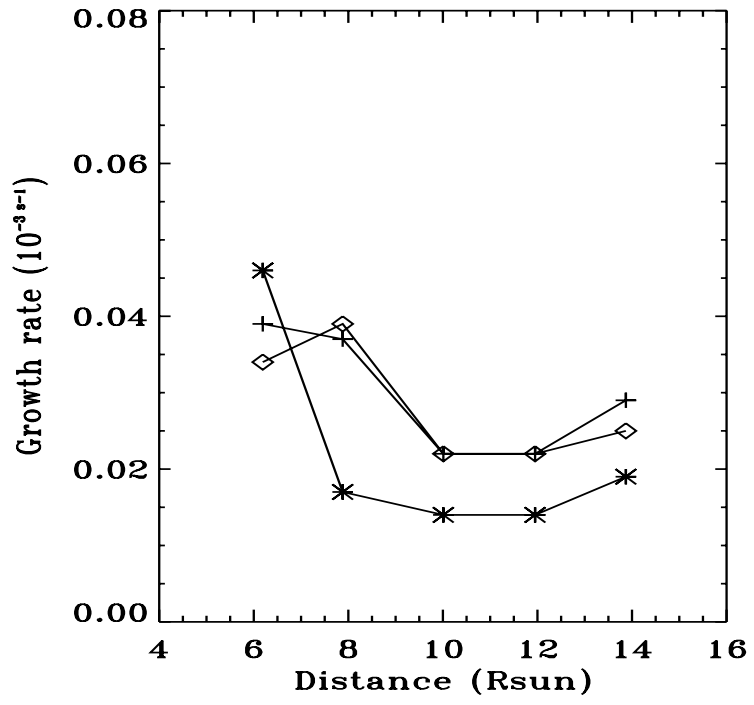

Fig. 7. Growth rate obtained from the $a-v$ graphs by forming five groups of CMEs according to their mean radial distance: asterisk for a-Vlinear, plus for a-Vinitial, diamond for a-Vextrap (please see text for more details).

speed with acceleration and growth rate. The importance of the initial speed for propagation in the LASCO range is demonstrated by this study. Using the linear speed-acceleration data, Vrsnak et al. (2004) obtained correlation coefficients for acceleration-speed plot for different subsets (classified according to number of height measurements and CME speed at $3 R_{\odot}$, $5 R_{\odot}$ using quadratic fit on height-time data and linear speed). They obtained the largest correlation coefficient between acceleration and speed for $n 6 v 3$ and $n 4 v 3$ sets, where $n 4$ and $n 6$ mean the number of height measurements 4 and 6, respectively, and v3 means the speed at $3 R_{\odot}$.

In addition, we isolated limb CMEs, which occurred at longitudes beyond 60 degrees. To do this, we first identified the locations of all the $117 \mathrm{CMEs}$ using known flares (from solar geophysical data), EUV dimming, and eruptive prominences. We found 59 events were behind the limb, 39 were limb CMEs, and the remaining were disk events. The $39 \mathrm{limb}$ CMEs are not subject to projection effects. For the limb CMEs, the range of linear speed is 251-902 $\mathrm{km} \mathrm{s}^{-1}$ with an average of $515 \mathrm{~km} \mathrm{~s}^{-1}$; the range of initial speed is $28-1208 \mathrm{~km} \mathrm{~s}^{-1}$ with an average of $442 \mathrm{~km} \mathrm{~s}^{-1}$. More interestingly, the correlation of initial speed with acceleration improved significantly to $60 \%$ from the earlier value of $53 \%$. However, no correlation was found between linear speed and acceleration and growth rate.

It can be pointed out that the correlation between growth rate and speed is simply a reflection of the equation we used to calculate $\mathrm{m}$. If $v(h)=m h+v_{\mathrm{o}}$ then $m=\left(v-v_{\mathrm{o}}\right) / h$. Given that $h \gg v \sim v_{\mathrm{o}}$, it would seem that $\mathrm{h}$ is the governing parameter in
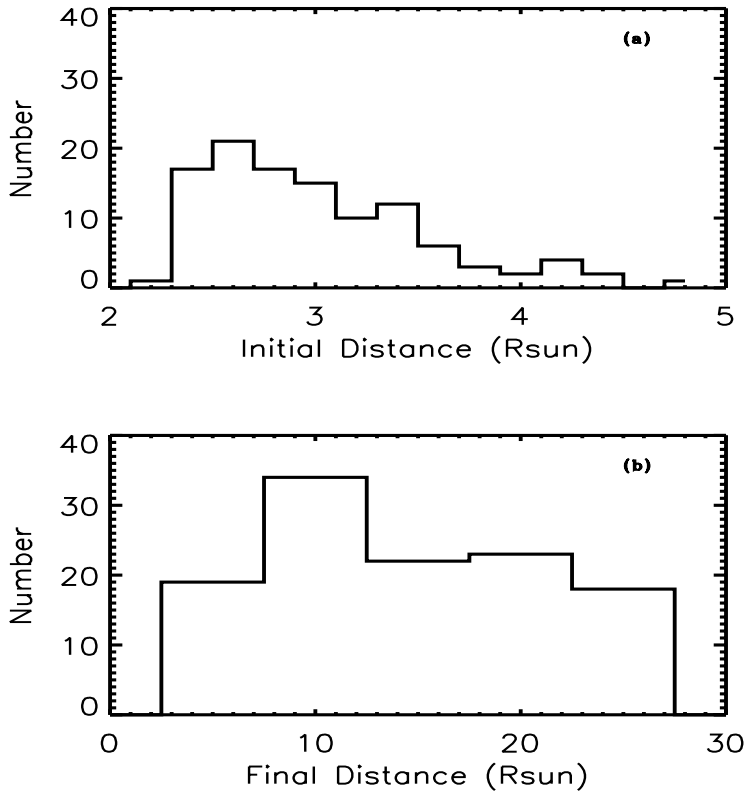

Fig. 8. Distributions of a) initial observed distance in the LASCO range, b) final observed distance in the LASCO range.

determining $m$, but $v=\mathrm{d} h / \mathrm{d} t$. It would seem that, in an indirect way, the parameters $m$ and $v_{\mathrm{o}}$ may be mathematically related. However, there is no such reflection in the correlation for two more cases, between (i) growth rate and initial speed; and between (ii) growth rate and linear speed.

In addition, we examined the final observed distance (FOD) of these CMEs in the LASCO FOV. The distributions of initial and final observed distances are shown in Fig. 8. These two limits are the first and last heights at which the brightness of the CMEs can be distinguished from the background in the LASCO FOV. As seen in this figure, while the probability of first detection of CMEs decreases exponentially between $2-5 R_{\odot}$, the distribution of FOD is nearly constant up to nearly $30 R_{\odot}$ except for a peak around $10 R_{\odot}$. That is, a significant number of CMEs (35/116) reach only around $10 R_{\odot}$ or they cannot be traced in SOHO/LASCO after this distance (Yashiro et al. 2004). The FOD distribution present in this study contrasts to previous results. For example, Gopalswamy (2004) found that FOD has a second peak beyond $20 R_{\odot}$, but these are due to fast and wide CMEs such as halo CMEs. Such fast and wide CMEs were excluded from our sample again due to the selection criterion, so we do not see them in our plot. The FOD distribution has an exponential trend for all CMEs, non-halo CMEs, and halo CMEs reported by Lara et al. (2006). However, a similar peak was reported around $5-10 R_{\odot}$ by them in the case of partial-halo CMEs. 
(a)

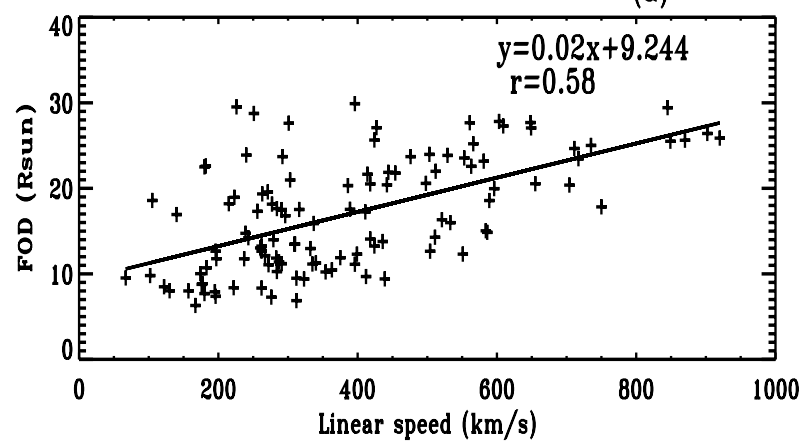

(b)

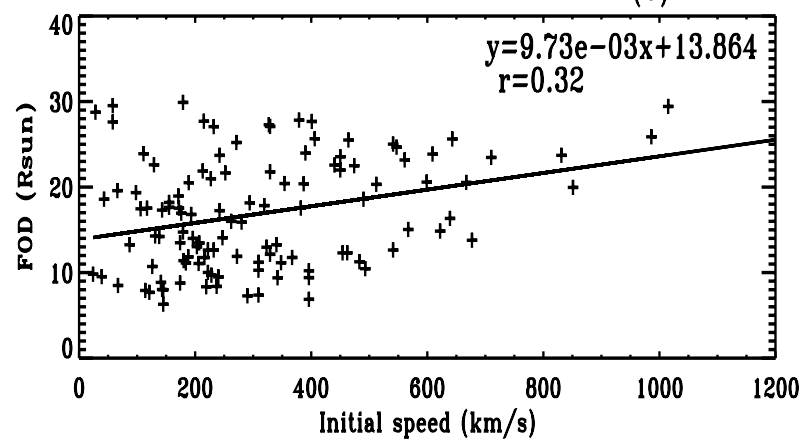

(c)

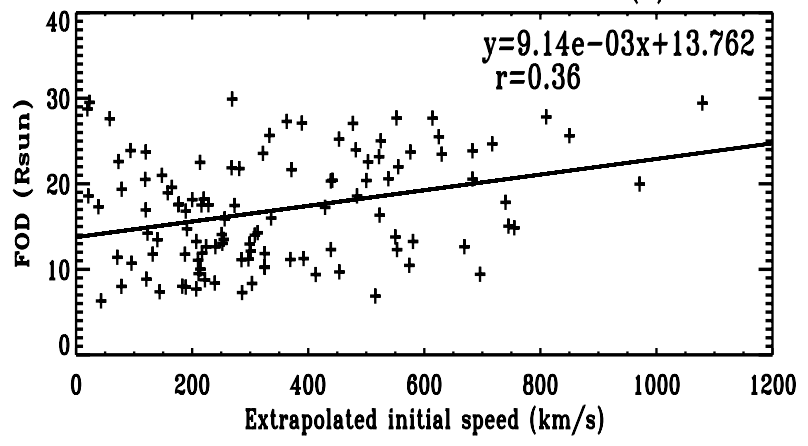

Fig. 9. Correlation between the final observed distance (FOD) with a) linear speed, b) initial speed, and c) extrapolated initial speed.

When we studied the correlation of FOD with the different speeds, we found a better correlation in the case of linear speed than in the cases of initial speeds (see Fig. 9). The poor correlation implies that the final height reached by the CME does not depend on the initial speed of the CMEs, but instead depends on a combination of propelling and retarding forces acting on the CMEs over the whole height range.

\section{Conclusion}

From the height-time data of 307 CMEs observed with SOHO/LASCO during January-February 2005, we used only 116 events for which there were at least 10 height-time measurements in the LASCO C2 and C3 range and studied the speed evolution profile of the CMEs. With these cataloged data, we obtained the initial speed from the first pair of height-time measurement and speed-distance profile of all the CMEs. Then the speed-distance profile was fitted with a linear fit $(v(h)=$ $\left.m h+v_{\mathrm{o}}\right)$. Using it, we obtained the growth rate $(m=\mathrm{d} v / \mathrm{d} h)$ and extrapolated initial speed $\left(v_{0}\right)$. The initial speed and the linear speed were correlated with other physical characteristics of the CMEs such as acceleration, growth rate, and final observed distance. The following results were obtained from this analysis. (i) The initial speed obtained from the first two data is in the range $24-1208 \mathrm{~km} \mathrm{~s}^{-1}$, which is nearly similar to the linear speed $\left(67-920 \mathrm{~km} \mathrm{~s}^{-1}\right)$ obtained from the least square fit through the entire $h-t$ data set. However, the mean initial speed $\left(320 \mathrm{~km} \mathrm{~s}^{-1}\right)$ is slightly lower than for the mean linear speed $\left(380 \mathrm{~km} \mathrm{~s}^{-1}\right)$. (ii) Moreover, the initial speed or extrapolated speed is more correlated with acceleration and growth rate than the linear speed. (iii) Nearly two thirds of the events (74/116) are accelerating; and (iv) the growth rate is within the acceptable range -0.058 to $0.061 \times 10^{-3} \mathrm{~s}^{-1}$ and it decreases with the height. (v) The final height reached by the CMEs depends weakly on the initial speed but strongly on the linear speed.

The above results suggest that the initial speed depends more on acceleration and the growth rate. The initial speed of the CMEs close to the Sun in the LASCO FOV and the coupling of CME with the solar wind are therefore also important in deciding propagation of CMEs in the upper corona.

Acknowledgements. We sincerely thank the referee for his or her comments and suggestions that improved the quality of this paper. This work has been supported by the Korea Research Foundation (KRF-2005-070-C00059), "Space Weather Center Project" of KASI and the KASI basic research fund. The author AS especially thanks Seiji Yashiro and Sachiko Akiama for providing the source locations of the events presented in this paper. The CME catalog we have used is generated and maintained by the Center for Solar Physics and Space Weather, The Catholic University of America in cooperation with the Naval Research Laboratory and NASA. SOHO is a project of international cooperation between ESA and NASA.

\section{References}

Cargill, P. J. 2004, Sol. Phys., 221, 135

Cho, K.-S., et al. 2005, J. Geophys. Res., 110

Gopalswamy, N. 2004, A Global Picture of CMEs in the Inner Heliosphere, in The Sun the Heliosphere As an Integrated System, ed. G. Poletto, \& S. Suess (New York: Springer), 201

Gopalswamy, N., et al. 2000, Geophys. Res. Lett., 27, 145

Gopalswamy, N., Lara, A., Yashiro, S., Kaiser, M. L., \& Howard, R. A. 2001a, J. Geophys. Res., 106, 29207

Gopalswamy, N., Yashiro, S., Kaiser, M. L., Howard, R. A., \& Bougeret, J.-L. 2001b, J. Geophys. Res., 106, 29219

Gopalswamy, N., Yashiro, S., Krucker, S., Stenborg, G., \& Howard, R. A. 2004, J. Geophys. Res., 109, 12105

Gosling, J. T. 1993, J. Geophys. Res., 98, 18937

Lara, A., Gopalswamy, N., Xie, H., et al. 2006, J. Geophys. Res., 111, A06107 Lin, J., Mancuso, S., \& Vourlidas, A. 2006, ApJ, 649, 1110

Lindsay, G. M., Luhmann, J. G., Russell, C. T., \& Gosling, J. T. 1999, J. Geophys. Res., 104, 12515

Vrsnak, B. 2001a, J. Geophys. Res., 106, A11, 25249

Vrsnak, B. 2001b, Sol. Phys., 202, 173

Vrsnak, B., \& Gopalswamy, N. 2002, J. Geophys. Res., 107

Vrsnak, B., Ruzdjak, D., Sudar, D., \& Gopalswamy, N. 2004, A\&A, 423, 717

Wood, B. E., Karovska, M., Chen, J., et al. 1999, ApJ, 512, 484

Yashiro, S., Gopalswamy, N., Michalek, G., et al. 2004, J. Geophys. Res., 109

Zhang, J., \& Dere, K. P. 2006, ApJ, 649, 1100.

Zhang, J., Dere, K. P., Howard, R. A., Kundu, M. R., \& White, S. M. 2001, ApJ, 559,452 